\title{
Identification des variétés de pommiers par l'enzyme phosphatase acide, son application à l'étude de la pollinisation des vergers
}

\author{
J.P. Torre Grossa
}

Station de Zoologie et d'Apidologie, INRA Avignon, 84140 Montfavet, France

(reçu le 20 septembre 1988, accepté le 10 février 1989)

Résumé - Le marqueur enzymatique phosphatase acide (ACP1) permet d'identifier les variétés de pommiers. L'analyse de pépins, issus de croisements variétaux ou d'autofécondations, montre que la répartition des allèles ACP1 est conforme aux proportions mendéliennes. Cette conformité permet de proposer une interprétation de l'origine incertaine de pépins issus d'une parcelle de «Golden Delicious" (faiblement autocompatible), pollinisée par des bouquets de "Starkrimson", ces deux variétés possédant un allèle commun. Cette méthode de marquage sera utilisée pour étudier la dispersion des pollens dans les vergers et la distribution de leurs vecteurs, les abeilles domestiques.

dispersion pollinique - identification de cultivar - marqueur enzymatique - phosphatase acide - Malus domestica

\section{Introduction}

L'étude de la distribution, en verger de pommiers, des abeilles domestiques (Apis mellifica F.) s'est heurtée, jusqu'à ces dernières années, à l'absence d'une méthode prenant en compte la totalité d'une population de butineuses. Williams et al. (1979) puis Lambert (1984), furent les premiers à envisager l'emploi de marqueurs génétiques, transmissibles par le pollen, dans le but d'étudier la dispersion et le comportement des abeilles pollinisatrices en vergers de pommiers. Le premier utilisa le caractère anthocyané de la variété «Baskatong" et le second, celui de la résistance à la tavelure de la variété
"Evereste perpétue», toutes deux étant des variétés botaniques. Le principe est d'approvisionner en pollen marqué, un dispositif placé à l'entrée des ruches. En sortant, les abeilles se chargent de pollen qu'elles dispersent ensuite dans une parcelle plantée, selon le cas, d'une variété non anthocyanée ou non résistante à la tavelure. La distribution des abeilles est cartographiée par l'analyse de la dispersion des marqueurs, observée sur des plantules issues d'un échantillon de pépins représentatif du verger. L'utilisation de tels marqueurs présente quelques inconvénients, tels que leur caractère polygénique, qui ne permet pas une identification fidèle de l'origine des pollens 
fécondants et la perte d'information, due à l'inévitable part de pépins ne germant pas, ou n'atteignant pas le stade plantule. Shen et al. (1981), ont étudié un modèle de pollinisation dans une pépinière de pins et Glaszmann et al. (1982), les flux géniques entre sous-espèces de Dactyles. Pour reconnaître l'origine des pollens fécondants, ces auteurs ont employé des marqueurs enzymatiques révélés par électrophorèse.

Le présent travail consiste dans l'identification de variétés de pommes en utilisant le polymorphisme du système enzymatique de la phosphatase acide (ACP1). Par plusieurs croisements et une autofécondation contrôlés de variétés, nous avons voulu tester, au niveau de la descendance (les pépins), l'hypothèse d'une conformité de la répartition génétique des allèles ACP1 à la loi mendélienne. Nous avons, par ailleurs, analysé la dispersion du pollen, assuré par les abeilles domestiques, à l'intérieur d'une parcelle d'un hectare, associant une variété commerciale faiblement autocompatible (Golden Delicious) à une variété pollinisatrice (Starkrimson). Ces deux variétés présentent l'inconvénient de posséder un allèle en commun, se traduisant par la difficulté de reconnaître l'origine du parent mâle de certains pépins. Deux méthodes correctives sont discutées.

\section{Matériels et Méthodes}

\section{Techniques électrophorétiques utilisées}

Le matériel végétal analysé est le pépin, porteur d'une moitié des parentés mâle et femelle (pour les variétés diploïdes). Toutes les parties du pépin peuvent être analysées sauf les téguments. Cependant, la radicule présente l'avan- tage d'une révélation enzymatique d'une grande finesse, exempte de taches susceptibles de gêner l'interprétation des résultats.

La phosphatase acide est extraite, de la radicule broyée à $0^{\circ} \mathrm{C}$, dans $50 \mu$ d'un tampon constitué de Tris $\mathrm{HCl}(0,2 \mathrm{M}$ Tris, $\mathrm{pH} 7,6)$, contenant $1 \%$ de polyéthylèneglycol 20000 et $10 \%$ de saccharose. Lors du broyage sont rajoutés $10 \mu \mathrm{l}$ d'une solution de rouge de phénol, permettant de visualiser le front de migration. Le broyat est centrifugé à $12500 \mathrm{~g}$ durant 15 $\min$ à $4^{\circ} \mathrm{C}$.

La séparation s'effectue par une électrophorèse sur gel de polyacrylamide à $8 \%$, en migration verticale d'une durée de $4 \mathrm{~h}: 15 \mathrm{~min}$ à 150 $V$ et le reste à $300 \mathrm{~V}$. Le tampon de gel est un Tris Sulfate $(0,065 \mathrm{M}$ Tris, $\mathrm{pH} 8,4)$, et celui des cuves un Tris Borate $(0,065 \mathrm{M}$ Tris, $\mathrm{pH} 8,4)$.

Après la migration, le gel est préincubé à température ambiante durant $20 \mathrm{~min}$, dans un tampon Acétate $(0,01 \mathrm{M}, \mathrm{pH} 4)$. La phosphatase acide est révélée dans $100 \mathrm{ml}$ de ce même tampon, additionné de $100 \mathrm{mg}$ d' $\alpha$ Naphtyl Acid Phosphate et de $100 \mathrm{mg}$ de Fast Garnet GBC. Les gels sont fixés dans une solution MéthanolAcide acétique-Eau en proportion 5-1-5.

\section{Identification des variétés de pommes}

Elle résulte de l'analyse des radicules de pépins issus d'une pollinisation libre de 34 variétés diploïdes. Les pépins sont récoltés dans le dispositif multivariétal des collections du Centre Expérimental Horticole de Marsillargues (Hérault). Ce dispositif favorise un grand brassage génétique qui renforce la probabilité de rencontre d'allèles identiques chez les radicules des pépins issus d'une variété hétérozygote, permettant ainsi son identification. L'identité d'une variété homozygote est caractérisée par la présence constante, chez chacune des radicules analysées, d'un même allèle. L'échantillon le plus petit nécessaire à ces identifications est de 24 radicules, dans certains cas litigieux cet effectif doit être doublé.

Dans le but de tester l'expression des allèles marqueurs de la phosphatase acide, sont analysées les radicules de pépins, issues de croisements manuels entre deux variétés préalablement identifiées. Dans ce même but, sont analysées deux générations de pépins issus d'autofécondation. La première (12) pro- 
vient d'un arbre lui même issu d'une première autofécondation (I1) de la variété partiellement autocompatible "Golden Delicious» et la seconde (13) d'une autofécondation d'un arbre de deuxième génération (12).

\section{Application au champ}

En 1984, sur une exploitation située au Thor (Vaucluse) l'étude de la dispersion des pollens fut abordée par l'analyse enzymatique des radicules de pépins, issues d'un verger d' 1 hectare dont la variété commerciale est «Golden Delicious" (1 222 arbres). Cette parcelle est pollinisée par la variété «Starkrimson», présentée sous forme de bouquets suspendus aux arbres de «Golden Delicious" ( 1 bouquet de 5 rameaux tous les 4 arbres, soit 312 bouquets dans le verger). La pollinisation libre est effectuée en présence de 2 ruches. L'échantillon jugé représentatif est constitué de 120 arbres, répartis sur l'ensemble de la parcelle. Sur chacun de ces arbres sont cueillies, en partant de la base d'une branche charpentière, les 10 premières pommes rencontrées. Du mélange des pépins de ces 10 pommes, il en est prélevé 24 destinés à l'analyse enzymatique.

\section{Résultats}

\section{Description de l'enzyme}

La phosphatase acide, se caractérise par 2 loci (Fig. 1) : l'ACP2, codant pour les bandes les plus lentes, est monomorphe et I'ACP1, codant pour les bandes les plus rapides, est polymorphe. Chez ce dernier, 6 allèles sont reconnus, dont 4 servent à l'identification des variétés des pommiers. Ils sont dénommés, du plus rapide au plus lent, $A C P 1 a, A C P 1 b$, $A C P 1 c$ et ACP1d. La présence, chez les combinaisons hétérozygotes, de 2 uniques bandes révèle le caractère monomérique de cette enzyme.

\section{Identification des variétés}

Le Tableau I réunit les variétés analysées et leur identité ACP1. Seuls manquent, les homozygotes $c c$ et bb. Le Tableau II

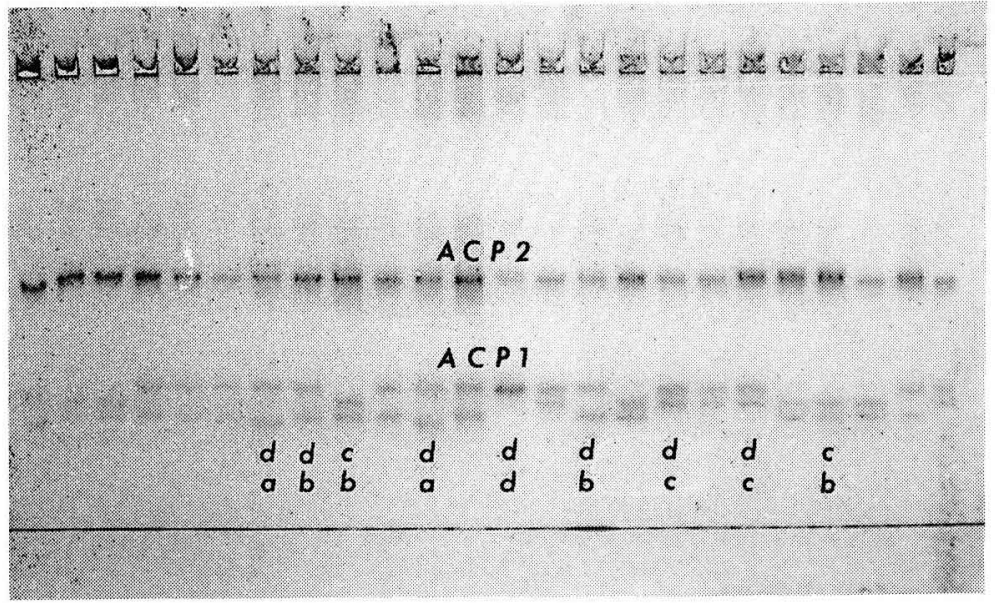

Fig. 1. Photographie d'un gel polyacrylamide représentant les différents niveaux d'allèles de l'ACP1. 
Tableau I. Identification ACP1 de variétés de pommiers.

\begin{tabular}{|c|c|c|c|c|c|}
\hline Variétés & $\begin{array}{l}\text { Allèles } \\
\text { ACP1 }\end{array}$ & Variété & $\begin{array}{l}\text { Allèles } \\
\text { ACP1 }\end{array}$ & Variété & $\begin{array}{l}\text { Allèles } \\
\text { ACP1 }\end{array}$ \\
\hline $\begin{array}{l}\text { Golden Delicious } \\
\quad+\text { mutants } \\
\text { Vista Bella } \\
\text { Rosu de Cluj } \\
\text { Idared } \\
\text { Granny Smith } \\
\text { Cardinal } \\
\text { Red Delicious } \\
\quad+\text { mutants } \\
\text { Reine des Reinettes } \\
\quad \text { + mutants }\end{array}$ & $\begin{array}{l}c d \\
\text { aa } \\
\text { ad } \\
\text { aa } \\
\text { bc } \\
\text { bc } \\
\text { bd } \\
\text { dd }\end{array}$ & $\begin{array}{l}\text { Cox's Orange Pippin } \\
\text { Alkmene } \\
\text { Worcester Pearmain } \\
\text { Barina } \\
\text { Jerseymac } \\
\text { Florina } \\
\text { Delcorf } \\
\text { Prima } \\
\text { Gala } \\
\text { Chantecler } \\
\text { Cloden }\end{array}$ & $\begin{array}{l}\text { dd } \\
\text { dd } \\
\text { dd } \\
\text { dd } \\
a b \\
a b \\
\text { ad } \\
\text { ad } \\
c d \\
c d \\
c d\end{array}$ & $\begin{array}{l}\text { Ozark Gold } \\
\text { Jonathan } \\
\quad+\text { mutants } \\
\text { Merrigold } \\
\text { Red Winesap } \\
\text { Deljeni } \\
\text { Dalicat } \\
\text { Newton Pippin } \\
\text { Calville Blanc } \\
\text { Coquette }\end{array}$ & $\begin{array}{l}\text { bd } \\
\text { ab } \\
a c \\
a b \\
a b \\
c d \\
a d \\
c d \\
\text { bd }\end{array}$ \\
\hline
\end{tabular}

des parents est connue. L'ACP1 de ces variétés est conforme aux possibilités de combinaisons résultant des croisements parentaux, exceptée la variété «Malling Kent».

\section{Pollinisations contrôlée et manuelle}

Le text de $\chi^{2}$ effectué, tant en autopollinisation de "Golden Delicious" (Tabl. III) qu'en pollinisation croisée entre "Granny Smith" et "Akane», "Idared» et "Granny
Smith», "Granny Smith» et "Golden Delicious" et «ldared" et «Akane» (Tabl. IV, $\mathrm{V}, \mathrm{VI}$ et VII), ne montre aucune différence significative entre les résultats attendus et observés. II souligne la conformité de la répartition des allèles à la loi génétique mendélienne.

\section{Pollinisation libre}

Les résultats du Tableau VIII révèlent l'inconvénient, dans une expérimentation au

Tableau II. Identification ACP1 de variétés de pommiers et de leurs parents.

\begin{tabular}{|c|c|c|c|c|c|}
\hline Variétés & $\begin{array}{l}\text { Allèles } \\
\text { ACP1 }\end{array}$ & Parent femelle & $\begin{array}{l}\text { Allèles } \\
\text { ACP1 }\end{array}$ & Parent mâle & $\begin{array}{l}\text { Allèles } \\
\text { ACP1 }\end{array}$ \\
\hline $\begin{array}{l}\text { Melrose } \\
\text { Hatsuaki } \\
\text { Grifer } \\
\text { Akane } \\
\text { Inra F.19.37 } \\
\text { Malling Kent* }\end{array}$ & $\begin{array}{l}a b \\
b c \\
b c \\
b d \\
\text { ac } \\
\text { dd }\end{array}$ & $\begin{array}{l}\text { Jonathan } \\
\text { Jonathan } \\
\text { Golden Delicious } \\
\text { Jonathan } \\
\text { Golden Delicious } \\
\text { Cox's Orange Pippin }\end{array}$ & $\begin{array}{l}a b \\
a b \\
c d \\
a b \\
c d \\
d d\end{array}$ & $\begin{array}{l}\text { Red Delicious } \\
\text { Golden Delicious } \\
\text { Jonathan } \\
\text { Worcester Paermain } \\
\text { Jonathan } \\
\text { Jonathan }\end{array}$ & $\begin{array}{l}\text { bd } \\
c d \\
a b \\
d d \\
a b \\
a b\end{array}$ \\
\hline
\end{tabular}

\footnotetext{
* Non concordance de l'identité de la variété Malling Kent avec celle de ses parents.
} 
Tableau III. Analyse ACP1 de pépins d'origine autofécondée. 12 : pépins provenant d'un arbre luimême issu d'une première autofécondation (11) de Golden Delicious. 13 : pépins provenant d'un arbre de la génération 12.

\section{Variété Descendances Golden Delicious}

\begin{tabular}{|c|c|c|c|c|c|}
\hline $\begin{array}{l}\text { Identité ACP1 } \\
\text { des parents }\end{array}$ & $c d$ & $x$ & $c d$ & $\chi^{2}$ & (DDL2) \\
\hline Proportions théoriques & $1 / 4$ & $2 / 4$ & $1 / 4$ & & \\
\hline Combinaisons & $\mathrm{cc}$ & $\mathrm{cd}$ & $d d$ & & \\
\hline$*$ Effectifs $\mathrm{l}_{2}$ & 22 & 53 & 21 & 1,07 & NS \\
\hline ** Effectifs $\mathrm{I}_{3}$ & 26 & 46 & 24 & 0,25 & NS \\
\hline
\end{tabular}

* Pépins $\left.\right|_{2}=I_{1} \times l_{1}$.

**épins $I_{3}=I_{2} \times I_{2}$.

Tableau IV. Analyse ACP1 de 115 pépins issus de pollinisation croisée, contrôlée, entre les variétés Granny Smith (?) et Akane ( ()$\left.^{\prime}\right)$.
Variétés
Granny Smith (?) x Akane (o')

\begin{tabular}{llllllll}
$\begin{array}{l}\text { Identité } A C P 1 \\
\text { des parents }\end{array}$ & & $b c$ & $x$ & $b d$ & & $\chi 2$ & DDL1 \\
\cline { 1 - 4 } & & & & & & & \\
Proportions théoriques & $1 / 4$ & $1 / 4$ & $1 / 4$ & $1 / 4$ & & \\
Combinaisons & bb & bd & bc & cd & & \\
Effectifs & 27 & 32 & 29 & 27 & 0,48 & NS \\
\hline
\end{tabular}

Tableau V. Analyse ACP1 de 213 pépins issus de pollinisation croisée, contrôlée, entre les variétés Idared ( $(9)$ et Granny Smith $\left(6^{7}\right)$.

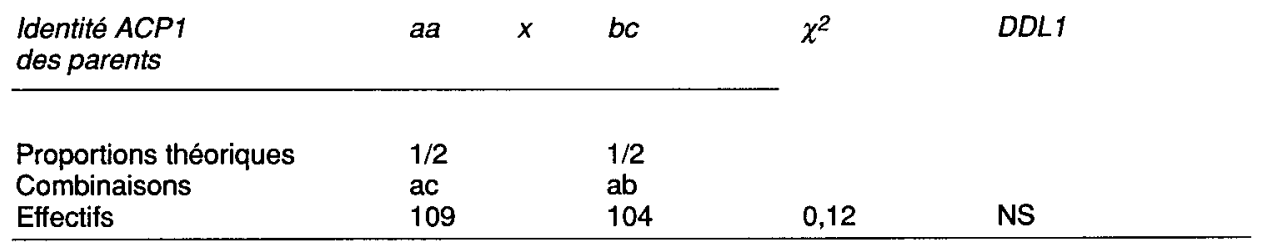


Tableau VI. Analyse ACP1 de 69 pépins issus de pollinisation croisée, contrôlée, entre les variétés Granny Smith ( $\left(\right.$ ) et Golden Delicious $\left(\sigma^{\circ}\right)$.

Variétés Granny Smith ( 7$) \times$ Golden $D\left(0^{7}\right)$

\begin{tabular}{|c|c|c|c|c|c|c|}
\hline $\begin{array}{l}\text { Identité } A C P 1 \\
\text { des parents }\end{array}$ & $b c$ & $x$ & $c d$ & & $x^{2}$ & $D D L 1$ \\
\hline $\begin{array}{l}\text { Proportions théoriques } \\
\text { Combinaisons } \\
\text { Effectifs }\end{array}$ & $\begin{array}{l}1 / 4 \\
b c \\
16\end{array}$ & $\begin{array}{l}1 / 4 \\
\text { bd } \\
15\end{array}$ & $\begin{array}{l}1 / 4 \\
c c \\
17\end{array}$ & $\begin{array}{l}1 / 4 \\
\mathrm{~cd} \\
21\end{array}$ & 1,20 & NS \\
\hline
\end{tabular}

champ, d'associer à une variété faiblement autocompatible, telle que "Golden Delicious" (cd), une variété poilinisatrice «Starkrimson» (bd) possédant un allèle identique, I'ACP1d. En effet, parmi les combinaisons cd et dd, certaines sont issues de pollinisation croisée et d'autres d'auto-pollinisation. En l'état, il apparaît difficile de reconnaître la part de deux pollinisations. Cependant, les résultats précédents permettent deux tentatives d'interprétation. Selon les Tableaux IV à VII, il devrait y avoir autant de bc et bd (pollinisation croisée reconnue) que de cd et dd. En fait, l'effectif théorique attribué à la pollinisation croisée $(b c+b d) \times 2=2888$ pépins) est supérieur à celui de l'échantillon (2 869 pépins) et donc, ne permet pas de prendre en considération l'autofécondation effective. Selon le Tableau III, il devrait y avoir, pour $1 / 4$ de cc d'origine autopollinisée reconnue (88 pépins), 2/4 de cd et $1 / 4$ de dd de même origine. Le taux de cd et dd issus d'autofécondation étant ainsi reconnu, il est possible, par différence, de connaître le taux de cd et dd issus d'une fécondation croisée. Ainsi traités, les résultats (Tableau IX) attribuent à la pollinisation croisée des effectifs cd et dd non significativement différents, cependant leur somme est inférieure à celle des bc + bd.

Tableau VII. Analyse ACP1 de 207 pépins issus de pollinisation croisée, contrôlée, entre les variétés Idared (o) et Akane (0).

Variétés Idared (o) X Akane (0)

\begin{tabular}{|c|c|c|c|c|c|}
\hline $\begin{array}{l}\text { Identité } A C P 1 \\
\text { des parents }\end{array}$ & $a a$ & $x$ & $b d$ & $\chi^{2}$ & $D D L 1$ \\
\hline $\begin{array}{l}\text { Proportions } \\
\text { Combinaisons } \\
\text { Effectifs }\end{array}$ & $\begin{array}{l}1 / 2 \\
a b \\
106\end{array}$ & & $\begin{array}{l}1 / 2 \\
\text { ad } \\
101\end{array}$ & 0,12 & NS \\
\hline
\end{tabular}


Tableau VIII. Analyse ACP1 d'un échantillon de 2869 pépins, issus d'une parcelle d'1 hectare de Golden Delicious, pollinisée par des bouquets suspendus de Starkrimson. " Combinaisons reconnues comme issues d'une autopollinisation, d'une pollinisation croisée ou incertaine.

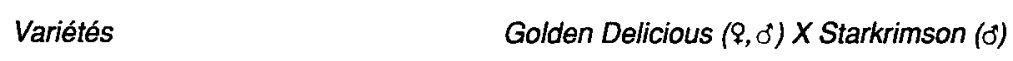

\begin{tabular}{|c|c|c|c|}
\hline $\begin{array}{l}\text { Identité } A C P 1 \\
\text { des parents }\end{array}$ & $c d$ & $x$ & $b d$ \\
\hline Combinaisons & $\begin{array}{l}\text { auto* (cd x cd) } \\
\text { cc }\end{array}$ & $\begin{array}{l}\text { incertaines* } \\
\text { cd dd }\end{array}$ & $\begin{array}{l}\text { croisées* }(c d x \text { bd }) \\
\text { bc bd }\end{array}$ \\
\hline Effectifs & 88 & $744 \quad 593$ & $732 \quad 712$ \\
\hline Pourcentage & 3,07 & $\begin{array}{c}25,93 \quad 20,67 \\
46,60\end{array}$ & $\begin{array}{c}25,50 \quad 24,82 \\
50,33\end{array}$ \\
\hline
\end{tabular}

Tableau iX. Données corrigées affectant les pépins d'origine incertaine à une autopollinisation ou une pollinisation croisée et déduction du taux de fécondation croisée et d'autofécondation rencontrées dans la parcelle. * Combinaisons attribuées à une autopollinisation de Golden Delicious ou à une pollinisation croisée par Starkrimson.

\begin{tabular}{|c|c|c|c|c|c|c|c|}
\hline \multirow[b]{2}{*}{ Combinaisons } & \multicolumn{3}{|c|}{ auto* $(\mathrm{cd} \times \mathrm{cd})$} & \multicolumn{4}{|c|}{ croisées ${ }^{*}(c d x$ bd) } \\
\hline & $\mathrm{cc}$ & $\mathrm{cd}$ & dd & cd & dd & $b c$ & bd \\
\hline Effectifs & 88 & 176 & 88 & 568 & 505 & 732 & 712 \\
\hline $\begin{array}{l}\text { Effectifs } \\
\%\end{array}$ & 3,07 & $\begin{array}{r}6,14 \\
12,27\end{array}$ & 3,07 & 19,80 & $\begin{array}{r}17,60 \\
8\end{array}$ & 25,50 & 24,82 \\
\hline
\end{tabular}

\section{Discussion - Conclusion}

Le marqueur enzymatique phosphatase acide a permis l'identification de 34 variétés diploïdes de pommes à couteau. Parmi les 10 identités ACP1 possibles, seules sont absentes les bb et les cc. Cependant, bien qu'absentes du Tableau I, les variétés ornementales «Golden
Gem", "Malus Hilliéri», "M. Aldenhamensis», "Winter Gold" et «TNR 31 35" sont identifiées bb.

L'identité dd de la variété «Malling Kent" n'est pas conforme aux possibilités qu'offrent celles des parents ( «Cox's Orange Pippin», dd $x$ «Jonathan», $a b=$ ad ou bd). II est probable, comme l'ont remarqué Weeden et Lamb (1985) pour 
une variété lors d'identifications enzymatiques, que l'origine parentale mâle soit autre que "Jonathan".

Dans des conditions de pollinisation contrôlée, tant en autofécondation de la seule variété partiellement autocompatible "Golden Delicious" qu'en fécondation croisée des variétés "Granny Smith" et «ldared» auto-incompatibles, les fréquences des combinaisons alléliques sont conformes aux proportions mendéliennes. Ceci suggère une répartition équitable des allèles marqueurs chez les éléments sexués haploïdes, mais également, labsence de freins à leur association.

Lorsque la variété "Golden Delicious" et son pollinisateur "Starkrimson" possèdent un allèle commun, il est difficile, en l'état, de reconnaître l'origine de la pollinisation dont sont issus certains pépins. Grâce aux résultats précédents, il est logique d'attribuer, à ces pépins, la part due à une autopollinisation et à une pollinisation croisée. Cependant, seule l'une des deux méthodes utilisées (attribuant à l'autofécondation, pour une combinaison cc reconnue d'origine autofécondée, deux cd et une dd) prend en compte la totalité des combinaisons que peut exprimer, dans sa descendance, une telle association variétale. Si cette correction était adéquate, nos résultats démontreraient que :

- l'allopollen b de la variété "Starkrimson" a assuré sa demi-part de fécondation croisée $(50,33 \%$ de bc + bd). Par contre, son allopollen $d$ n'assurerait que $37,4 \%(c d+d d)$ de cette même fécondation;

- l'autofécondation serait essentiellement effectuée au détriment de l'allopollen d. Ce dernier, rentrant en concurrence avec les autopollens $c$ et $d$ de "Golden Delicious";

- le taux de fécondation croisée $(87,73 \%)$ est remarquable, si nous consi- dérons qu'il est assuré par le polien de 312 bouquets de 5 rameaux de "Starkrimson» suspendus dans un verger de 1222 arbres de «Golden Delicious».

II apparaît donc que le marqueur enzymatique des variétés de pommiers, la phosphatase acide, est un nouveau moyen d'étudier la pollinisation des vergers commerciaux. Ainsi depuis 1984, sur une parcelle d'un hectare de "Golden Delicious", située au Thor (Vaucluse), des expériences ont été menées dans le but d'analyser la dispersion des pollens, la distribution de leur vecteur, l'abeille domestique, et les conséquences agronomiques des dispositifs de pollinisation mis en place. Les résultats de ces travaux feront, prochainement, l'objet de publications.

II est cependant acquis que la seule variabilité de la phosphatase acide n'assure pas une parfaite reconnaissance de l'origine des pollens fécondants. Lors d'expérimentations en verger de «Golden Delicious", il faudra s'assurer de l'absence, dans l'environnement, d'allopollens ayant un allèle commun à cette variété. Dans l'avenir, il faudra rechercher d'autres enzymes marqueurs des variétés de pommiers qui, associés à l'ACP1, renforceront la faculté d'identifier les origines des pollens fécondants.

\section{Summary - Apple cultivar identifica-} tion by means of the enzyme acid phosphatase. Applying it to the study of orchard pollination. The aim of the present work was to identify an enzymatic marker in pollen that eventually facilitates a study of honeybee (Apis mellifera L.) movement in apple orchards. This was based on the idea, proposed by Williams et al. (1979) and Lambert (1984) whereby the origin of the fertilizing pollen carried by the pollinator could be identified by analy- 
sis of visual markers of apple (anthocyanin, scab resistance).

Electrophoretic analysis of the enzyme acid phosphatase, using polyacrylamide gel, showed that the ACP1 locus was characterised by 6 alleles, 4 of which were useful in identifying apple cultivars (Fig. 1). The markers, ranked from the fastest to the slowest ACP1, a, b, c and d, were used to identify 34 diploid cultivars of apple. Among the 10 possible allelic combinations, only the bb and cc homozygotes were not found. The cultivar "Malling Kent", supposed to result from a cross between "Cox's Orange Pippin» (dd) and «Jonathan» (ab), was a dd homozygote which strongly suggests that "Jonathan" is not the pollen source for "Malling Kent".

Analysis of seeds obtained from the $I_{2}$ and $I_{3}$ generations of partially self-compatible cultivar "Golden Delicious", as well as those from controlled crosses between "Granny Smith x Akane", "Idared x Granny Smith», "Granny Smith x Golden Delicious" and "ldared x Akane", showed that allelic frequencies conformed with mendellian predictions.

When free pollination occurred in an experimental plot of the partially self-compatible "Golden Delicious" (cd) with a pollen supply of the outcrossing cultivar "Starkrimson" (bd) it was not possible, due to the common $d$ allele, to clearly identify the origin of cd and dd seeds. However, using the results obtained from the different controiled crosses, and the self-pollination of "Golden Delicious", it was possible to estimate the proportions resulting from self and cross-pollination. The $b$ allele from «Starkrimson" accounted for $50.3 \%$ of all bc and bd combinations compared with only $37.4 \%$ of the $d$ allele in the cd and dd combinations. Thus "Golden Delicious" self-pollination has a competitive effect on the "Starkrimson" d allele. The observed $87.7 \%$ cross-pollination is very high when one considers that there were 1222 "Golden Delicious" trees and only 315 groups of 5 "Starkrimson" branches.

The enzyme acid phosphatase appears to be a useful marker in future studies of pollen dispersal, as well as honeybee distribution, within commercial apple orchards. However, the addition of other enzymatic markers is desirable, thereby increasing our ability to discriminate between pollen sources.

pollen dispersion - cultivar identification - enzymatic marker - Malus domestica

\section{Zusammenfassung - Bestimmung von Apfelsorten durch das Enzym "saure Phosphatase" und dessen} Anwendung beim Studium der Bestäubung von Obstkulturen. Das Ziel dieser Arbeit war es, eine enzymatische Marke im Pollen zu bestimmen, die möglicherweise eine Untersuchung der Flugbewegungen von Honigbienen (Apis mellifera L.) in den Apfelanlagen erleichtert. Sie basiert auf einer Idee von Williams et al. (1979) und Lambert (1984), der zufolge der Ursprung des von den Bestäubern zur Befruchtung übertragenen Pollens durch die Analyse der sichtbaren Marken von Äpfeln (Anthozyanin, Pilze) bestimmt werden kann.

Die electrophoretische Analyse des Enzyms "saure Phosphatase" durch Polyacrylamid-Gel zeigte, daß der ACP1Locus durch 6 Allele charakterisiert war, von denen 4 für die Bestimmung von Apfelsorten benutzt werden konnten (Abb. 1). Die Marken, in der Reihenfolge a, b, c und $d$ vom schnelisten zum langsamsten ACP1, wurden zur Bestimmung von 34 
diploiden Apfelsorten benutzt. Von den 10 möglichen Allelkombinationen wurden nur die Homozygoten bb und $c c$ nicht gefunden. Die Sorte "Malling Kent", angeblich das Resultat einer Kreuzung von "Cox's Orange Pippin" (dd) und "Jonathan" (ab) war homozygot für dd, was sehr stark vermuten läßt, daß «Jonathan» nicht die Pollenquelle für «Malling Kent» ist.

Die Analyse der Samen von den $\mathrm{I}_{2}$ und $\mathrm{I}_{3}$ Generationen der teilweise selbstbefruchtenden Sorte "Golden Delicious" sowie von kontrollierten Kreuzungen zwischen "Granny Smith x Akane", «ldared x Granny Smith", "Granny Smith x Golden Delicious" und "ldared x Akane" zeigte, daß die Allelfrequenzen mit den Mendel-Voraussagen übereinstimmten.

Wenn in einem experimentellen Feld der teilweise selbstbestäubenden Sorte «Golden Delicious» (cd) freie Bestäubung mit einem Pollenangebot von der fremdbestäubenden Sorte «Starkrimson» (bd) erfolgte, war es wegen des gemeinsamen d-Allels nicht möglich, den Ursprung von cd- und dd-Samen zu bestimmen. Anhand der Resultate von verschiedenen kontrollierten Kreuzungen und der Selbstbestäubung von "Golden Delicious" war es jedoch möglich, die Anteile von Selbstund Kreuzbestäubungen abzuschätzen. Das b-Allel von «Starkrimson» war für $50,3 \%$ von allen bc- und bd-Kombinationen verantwortlich, verglichen mit nur $37,4 \%$ des d-Allels in den cd- und ddKombinationen. Demnach hat die Selbstbestäubung von «Goiden Delicious» einen Konkurrenzeffekt auf das d-Allel von «Starkrimson». Die beobachteten $87,7 \%$ Fremdbestäubungen sind sehr hoch wenn man bedenkt, daß 1222 «Golden Delicious»-Bäume nur 315 Gruppen von je 5 «Starkrimson»-Zweigen gegenüber-standen.
Das Enzym "saure Phosphatase" ist also offensichtlich eine brauchbare Marke für künftige Studien über die Ausbreitung des Pollens wie über die Verteilung der Bienen in kommerziellen Apfelanlagen. Es wäre jedoch die Einführung einer weiteren enzymatischen Marke wünschenswert, wodurch die Möglichkeiten, zwischen Pollenquellen zu unterscheiden, noch anstejgen würden.

\section{Pollenverteilung - Sortenbestimmung - enzymatische Marke - Malus domestica}

\section{Références}

Glaszmann J.C., Lumaret R. et Debussche M. (1982) Contrôle écologique du flux génique entre deux sous-espèces tétraploïdes de dactyles (Dactylis glomerata L.) : étude d'une zone de contact dans le Sud du Massif Central (France). Acta Oecol. / Oecol. Plant. 3 (17), 87100

Lambert B. (1984) Estimation de la répartition de la pollinisation en verger monovariétal de pommiers, grâce à l'utilisation d'un gène marqueur (résistance à la tavelure) et de distributeurs de pollen. Symposium International sur la Pollinisation, Versailles (France), 27-30 sept. 1983. Ed. INRA Pub., Les Colloques de I'INRA, $n^{\circ} 21,405-408$

Shen H.H., Rudin D. and Lindgren D. (1981) Study of pollination pattern in a scots pine seed orchard by means of isoenzyme analysis. Silvae Genet. 30 (1) 7-15

Weeden N.F. and Lamb R.C. (1985) Identification of apple by isozyme phenotypes. J. Am. Soc. Hortic. Sci. 110 (4) 509-515

Williams R.R., Church R.M., Wood D.E.S. \& Flood V.A. (1979) Use of an anthocyanin progeny marker to determine the value of hive pollen dispensers in apple orchards. J. Hortic. Sci. 54 (1), 75-78 